\title{
The Identification and Characterization of New Immunogenic Egg Components: Implications for Evaluation and Control of the Immunopathogenic T Cell Response in Schistosomiasis
}

\author{
Miguel J Stadecker ${ }^{+}$, Hector J Hernandez, Hiroko Asahi ${ }^{++}$
}

Department of Pathology, Tufts University School of Medicine, 136 Harrison Avenue, Boston, MA 02111, USA

In schistosomiasis, granuloma formation to parasite eggs signals the beginning of a chronic and potentially life-threatening disease. Granulomas are strictly mediated by CD4+T helper (Th) cells specific for egg antigens; however, the number and identity of these T cell-sensitizing molecules are largely unknown. We have used monoclonal $T$ cell reagents derived from egg-sensitized individuals as probes to track down, isolate and positively identify several egg antigens; this approach implicitly assures that the molecules of interest are T cell immunogens and, hence, potentially pathogenic. The best studied and most abundant egg component is the Sm-p40 antigen. Sm-p40 and its peptide 234-246 elicit a strikingly immunodominant Th-1-polarized response in $C 3 H$ and CBA mice, which are $H-2^{k}$ strains characterized by severe egg-induced immunopathology. Two additional recently described $T$ cell-sensitizing egg antigens are Schistosoma mansoni phosphoenolpyruvate carboxykinase (SmPEPCK) and thioredoxin peroxidase-1 (Sm-TPx-1). In contrast to Sm-p40, both of these molecules induce a more balanced Th-1/Th-2 response, and are relatively stronger antigens in C57BL/6 mice, which develop smaller egg granulomas. Importantly, Sm-p40 and Sm-PEPCK have demonstrated immunogenicity in humans. The findings in the murine model introduce the important notion that egg antigens can vary significantly in immunogenicity according to the host's genetic background. A better knowledge of the principal immunogenic egg components is necessary to determine whether the immune responses to certain antigens can serve as indicators or predictors of the form and severity of clinical disease, and to ascertain whether such responses can be manipulated for the purpose of reducing pathology.

Key words: schistosomiasis - T cell response - egg antigens - CD4 + T helper cells

Infection with Schistosoma mansoni causes hepatic granuloma formation and fibrosis in response to parasite eggs; however, the magnitude of resulting disease varies greatly, both in humans as well as in an experimental mouse model. In humans, clinical schistosomiasis ranges from a relatively benign and symptomless "intestinal" form to a less frequent but severe "hepato-splenic" form, characterized by hepatic fibrosis, portal hypertension, gastro-intestinal hemorrhage and death. In mice, the $\mathrm{CBA}$ and $\mathrm{C} 3 \mathrm{H}$ inbred strains develop sig-

Grant support from NIAID/NIH, USA, and from TDR/ WHO.

${ }^{+}$Corresponding author. Fax: 617-636.2990. E-mail: miguel.stadecker@tufts.edu

${ }^{++}$Present address: Department of Parasitology, National Institute of Infectious Diseases, 1-23-1, Toyama, Shinjuku-ku, Tokyo, Japan 162-8640

Received 14 May 2001

Accepted 25 July 2001 nificantly larger egg granulomas than the C57BL/6 (BL/6) and BALB/c strains.

Granuloma formation is dependent on, and mediated by, MHC class-II-restricted CD4+ T helper (Th) lymphocytes with specificities directed against egg antigens (Mathew \& Boros 1986, Hernandez et al. 1997a), and can occur in environments dominated by either Th-1-or Th-2-type cytokines (Pearce et al. 1991, Chikunguwo et al. 1991, Henderson et al. 1992, Wynn et al. 1993). Recent studies in humans have demonstrated that a Th-1-biased cytokine profile is preferentially associated with hepato-splenic schistosomiasis (Mwatha et al. 1998), whereas patients with the milder intestinal disease display elevated anti-inflammatory Th-2type cytokines, notably IL-10 (Araujo et al. 1996, Malaquias et al. 1997). In mice, a common initial Th-1 response to soluble schistosome egg antigens (SEA) switches to a mixed Th- $1 /$ Th-2 phenotype in the large granuloma-forming $\mathrm{C} 3 \mathrm{H}$ strain (Hernandez et al. 1998) and to an entirely Th-2 phenotype in the small granuloma-forming BL/6 strain (Stadecker \& Hernandez 1998). It has been surmised 
that severe pathology in schistosomiasis is due to a defective spontaneous down-regulation ("immunomodulation") of the pathogenic immune response, for which various mechanisms have been proposed.

Despite considerable advances in the understanding of the immunopathological basis of the granulomatous inflammation in schistosomiasis, the nature of most of the $T$ cell-sensitizing egg antigens remains largely unknown. This contrasts with the relatively more plentiful available information about schistosomular and/or adult worm antigens, of which several have been cloned and are currently considered as candidates for an anti-infection vaccine (Bergquist 1998). Egg antigens are host-immunogenic glycoproteins that are primarily, but not necessarily exclusively, associated with the egg stage, and include those that are actively secreted and those that are released after the egg's death and disintegration. In any case, these egg components become available for accessory cell processing and presentation to $\mathrm{T}$ cells, and their immunogenicity and pathogenicity will vary in accordance with genetically determined host factors, including the MHC haplotypes and the T cell repertoire.

For many years, numerous efforts have been made by several laboratories to elucidate the immunogens present in SEA preparations. More recently, egg components separated on the basis of their physical and chemical properties, have been tested functionally for their ability to stimulate lymphoproliferation and cytokine production in vitro (Harn et al. 1989, Lukacs \& Boros 1992). Even though these assays more closely ascertain their pathogenic potential, a precise identification of individual molecules has not been forthcoming. Figure represents an SDS-PAGE analysis that conveys an idea of the complexity of SEA, showing at least 30 components with apparent molecular masses ranging from $<25$ to $>200 \mathrm{kDa}$. It is improbable that all of these components are strong immunogens. However, several of them likely contribute to sensitizing the host for granuloma formation, although the precise number in each individual case is presently unknown.

\section{STRATEGY FOR THE IDENTIFICATION OF EGG ANTIGENS}

The basic approach taken by our laboratory is to test the separated egg components (Figure) for their ability to stimulate sets of SEA-specific monoclonal $\mathrm{T}$ cells (clones or hybridomas) derived from infected or sensitized mice. Each T cell probe recognizing one particular band is used to track down the stimulatory material through the necessary purification steps until partial sequences can lead to the identity and cloning of the antigen, either by search of existing databases or by reverse genetics. This approach implicitly assures that each of the selected molecules is a T cell immunogen with potential pathogenicity. T cell hybridomas and specific polyclonal CD4+ Th cells are then reused to verify the stimulatory capacity and MHC restriction of the recombinant antigens, and of the corresponding antigenic fragments and synthetic peptides, in pursuit of the localization of the immunodominant epitope(s). Egg antigens are typical "exogenous" antigens that are processed in accessory cell vesicular compartments, where selected peptides bind to the host's MHC class II molecules, which are then externalized and presented to specific CD4+ Th cells. The assessment of MHC class II restriction of a particular antigenic peptide is, therefore, an important step to determine its relative immunogencity in context with a given haplotype.

\section{THE MAJOR Sm-p40 EGG ANTIGEN}

Sm-p40 is clearly the most abundant egg component, representing approximately $10 \%$ of SEA (Figure, band 15). It was first reported as a family of

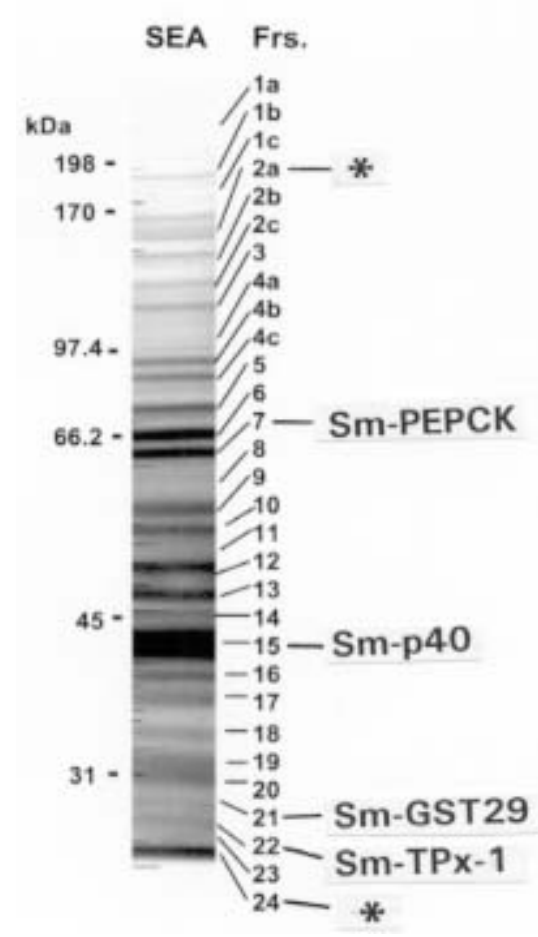

SDS-PAGE profile of SEA on immunoblots stained with amido black. 30 discernible bands (1a-24) are shown. The antigens described in this paper are indicated after the corresponding bands. An asterisk indicates components currently under investigation. Molecular weight marker standards are indicated on the left. Frs: fractions. 
near identical $40 \mathrm{kDa}$ proteins from eggs and miracidia, and the 354 amino acid sequence of one of the isoforms revealed substantial homology to alpha crystallins and Drosophila small heat shock proteins (Nene et al. 1986). We subsequently found that $\mathrm{Sm}-\mathrm{p} 40$ was the antigen recognized by cloned granulomagenic T cells (Chikunguwo et al. 1993), thereby documenting its pathogenicity; other laboratories described $\mathrm{Sm}-\mathrm{p} 40$ independently (Cao et al. 1993, Cai et al. 1996). The Sm-p40 antigen is strikingly immunodominant in the large granuloma-forming $\mathrm{H}-2^{\mathrm{k}}$ mouse strains such as $\mathrm{C} 3 \mathrm{H}$ and $\mathrm{CBA}$, as it was the sole antigen recognized by all members of a panel of clonally distinct SEA-specific T cell hybridomas (Hernandez et al. 1997b). In contrast, none of the SEA-specific T cell hybridomas derived from the small granuloma-forming BL/6 $\left(\mathrm{H}-2^{\mathrm{b}}\right)$ strain responded to Sm-p40. The Sm-p40 antigen has one immunodominant and at least two subdominant $\mathrm{T}$ cell epitopes (Chen \& Boros 1998, Hernandez et al. 1998). Close analysis of immunodominant $13 \mathrm{mer}$ peptide 234-246 PKSDNQIKAVPAS reveals D237 as the MHC class II (I-A ${ }^{\mathrm{k}}$-anchoring residue, and N238, Q239 and K241 as the principal TCR contact residues (Hernandez et al. 1999a). Unexpectedly, the Sm-p40 antigen, as well as its immunodominant 13mer peptide 234-246, elicit a mesenteric lymph node CD4+ T cell response that is strongly and lastingly biased in favor of the Th-1 type (Cai et al. 1996, Hernandez et al. 1998). This finding is significant because the mature response to unfractionated SEA is polarized towards the Th- 2 type (Pearce et al. 1991). Of equal significance is the distinct immunodominance of Sm-p40 in large granulomaforming $\mathrm{H}-2^{\mathrm{k}}$ mouse strains, as it raises the possibility of a correlation between an elevated immune response against Sm-p40 and the magnitude of disease. Preliminary data suggest that this is also the case in humans (personal observations), although more studies, currently in progress, are needed to prove this point.

\section{Sm-PEPCK}

The first egg antigen discovered as a result of a direct match between a $62 \mathrm{kDa}$ egg component (Figure, band 7) and a specific BL/6 T cell hybridoma is $S$. mansoni phosphoenolpyruvate carboxykinase (Sm-PEPCK), because an internal sequence from the isolated molecule exhibited identity or near identity with one found in this gluconeogenic enzyme of various other species (Asahi et al. 1999). SmPEPCK elicits a solid proliferative response in CD4+ Th cells from schistosome-infected mice, which, in contrast to Sm-p40, is significantly stronger in the $\mathrm{BL} / 6$ than in the CBA strain. Moreover, the relatively stronger cytokine response to Sm-PEPCK in $\mathrm{BL} / 6$ mice is of a more balanced Th- 1 and Th- 2 type.
The cloned, 626 amino acid recombinant Sm-PEPCK effectively stimulates CD4+ Th cells from schistosome-infected and SEA-immunized mice (Asahi et al. 2000); however, in three different infected mouse strains, this response is distinctly polarized towards the Th-1 type. Sm-PEPCK 12-mer peptide 398-409 (DKSKDPKAHPNS) contains the T cell epitope recognized by the identifying $\mathrm{T}$ cell hybridoma and by polyclonal CD4+ Th cells. Most importantly, SmPEPCK is also an immunogen in humans, as genetically influenced immune responses to Sm-PEPCK have been detected in schistosomiasis patients in Brazil (Bethony et al. 2000).

\section{Sm-TPx-1}

Another immunogenic schistosome egg component with an apparent molecular mass of $26 \mathrm{kDa}$ (Figure, band 22) was recently identified as thioredoxin peroxidase-1 (TPx-1), a member of a class of antioxidant proteins called peroxiredoxins (Williams et al. 2001). TPx-1 is secreted by the eggs and has been localized by immunohistochemical analysis to the region between the miracidium and the eggshell (von Lichtenberg's envelope). The native TPx-1 effectively stimulates proliferation and a mixed Th-1 and Th-2-type cytokine production by CD4+ Th cells from schistosome-infected BL/6 and CBA mice; by comparison, the responses to the recombinant TPX-1 are smaller and more polarized towards the Th-1 type. Like Sm-PEPCK, TPx-1 is a relatively stronger antigen than Sm-p40 in BL/6 mice, whereas in the CBA strain Sm-p40 elicits a stronger response than TPx-1. TPx-1 has also been found to stimulate a humoral response in BL/6, CBA and BALB/c mice; the most significant antibody production takes place following parasite oviposition, peaks after 9 weeks of infection and declines thereafter.

\section{OTHER EGG ANTIGENS}

Partial sequence analysis of a $29 \mathrm{kDa}$ egg component (Figure, band 21) recognized by a BL/6 T cell hybridoma has revealed homology to a previously reported isoform of glutathione-S-transferase (GST) (Tiu et al. 1988). Other egg components are currently being analyzed. These are indicated with asterisks in the Figure, and include moieties with molecular weights of $150-165 \mathrm{kDa}$ (band 2a) and 19 $\mathrm{kDa}$ (band 24); the availability of EST should facilitate their identification. Several additional bands shown in the Figure may be potentially immunogenic egg components that remain to be elucidated. At least one of these probably represents leucine aminopeptidase (Xu et al. 1990).

\section{DISCUSSION AND CONCLUSIONS}

The use of monoclonal $\mathrm{T}$ cell probes has made it possible to positively identify several schisto- 
some egg antigens. This strategy could eventually lead to the identification, in order of importance, of most egg immunogens, assuming that the chance of obtaining a $\mathrm{T}$ cell hybridoma for any given antigen is proportional to the frequency of T cell clones with specificities for it. Another advantage of this approach is that the isolated antigens are $\mathrm{T}$ cell immunogens and thus likely involved in granuloma formation, which is mediated by MHC class II-restricted CD4+ Th cells. These pathogenic T cells recognize peptide epitopes on the egg antigens, which is confirmed by their stimulation with recombinant antigens and synthetic peptides.

Of considerable interest is the observation that the isolated egg antigens stimulate significant CD4+ Th-1-type cytokine responses in CD4+ Th cells at a time of infection when the overall $\mathrm{T}$ cell response to SEA is inclined towards the Th-2 type. This is particularly true in the case of the Sm-p40 antigen. In turn, recombinant antigens stimulate even more Th-1-polarized responses than their natural counterparts. One plausible explanation for this phenomenon is that natural antigens are glycosylated and recombinant antigens are not. Several N- and O- glycans and glycolipids capable of inducing antibody responses have been described in the various stages of the schistosome life cycle, including the eggs (Cummings \& Nyame 1999), although the level of glycosylation of the various antigens, or the type of glycan(s) attached to them, have not been determined. For example, indications from its amino acid composition and relative electrophoretic mobility suggest that $\mathrm{Sm}$ p40 is only lightly glycosylated. Another possibility is that factors in SEA, structurally unrelated to these antigens, promote Th-2-type cytokine secretion. Regardless, it is likely that normal antibody responses to glycans contribute to the development of a beneficial host-protective Th-2 milieu; this notion stems from the observation that in Bless mice and in B7-1/B7-2 costimulatory moleculedeficient mice, which cannot mount normal antibody responses (Borriello et al. 1997), the hepatic immunopathology may be hightened while the Th2 response to SEA fails to materialize (Hernandez et al. 1999b, 1997c, Ferru et al. 1998).

In sum, the overall magnitude of immunopathology in schistosomiasis in any given individual could be construed as the product of a predominantly cellular, anti-peptide, Th-1-polarized and pathology-exacerbating response, counterbalanced by a predominantly humoral, anti-glycan, Th-2-polarized and pathology-attenuating response, both determined by the host's genetic background. Future studies will lead to a more systematic elucidation of the relevant peptide and glycan molecules and demonstrate if and how they can be subject to immunomanipulation for the ultimate purpose of reducing disease.

\section{REFERENCES}

Araujo MI, de Jesus AR, Bacellar O, Sabin E, Pearce E, Carvalho EM 1996. Evidence of a T helper type 2 activation in human schistosomiasis. Eur J Immunol 26: 1399-1403.

Asahi H, Hernandez HJ, Stadecker MJ 1999. A novel 62-kilodalton egg antigen from Schistosoma mansoni induces a potent CD4(+) $\mathrm{T}$ helper cell response in the C57BL/6 mouse. Infect Immun 67: 1729-1735.

Asahi H, Osman A, Cook RM, LoVerde PT, Stadecker MJ 2000. Schistosoma mansoni phosphoenolpyruvate carboxykinase, a novel egg antigen: immunological properties of the recombinant protein and identification of a T-cell epitope. Infect Immun 68: 3385-3393.

Bergquist NR 1998. Schistosomiasis vaccine development: progress and prospects. Mem Inst Oswaldo Cruz 93: 95-101.

Bethony J, Williams JT, Carvalho-Queiroz C, WilliamsBlangero S, Blangero J, Alves-Fraga L, Stadecker MJ, Soares-Silveira A, Correa-Oliveira R, LoVerde PT 2000. Genetic correlations between isotype responses and egg counts during Schistosoma mansoni infection. Am J Trop Med Hyg 62: 157.

Borriello F, Sethna MP, Boyd SD, Schweitzer AN, Tivol EA, Jacoby D, Strom TB, Simpson EM, Freeman GJ, Sharpe AH. 1997. B7-1 and B7-2 have overlapping, critical roles in immunoglobulin class switching and germinal center formation. Immunity 6: 303-313.

Cai Y, Langley J, Smith D, Boros D 1996. A cloned major Schistosoma mansoni egg antigen with homologies to small heat shock proteins elicits Th1 responsiveness. Infect Immun 64: 1750-1755.

Cao M, Chao H, Doughty B 1993. Cloning of a cDNA encoding an egg antigen homologue from Schistosoma mansoni. Mol Biochem Parasitol 58: 169-171.

Chen Y, Boros DL 1998. Identification of the immunodominant $\mathrm{T}$ cell epitope of $\mathrm{p} 38$, a major egg antigen, and characterization of the epitope-specific Th responsiveness during murine schistosomiasis mansoni. J Immunol 160: 5420-7.

Chikunguwo S, Kanazawa T, Dayal Y, Stadecker M 1991. The cell-mediated response to schistosomal antigens at the clonal level. In vivo functions of cloned murine egg antigen-specific CD4 + T helper type 1 lymphocytes. J Immunol 147: 3921-3925.

Chikunguwo S, Quinn S, Harn D, Stadecker M 1993. The cell-mediated response to schistosomal antigens at the clonal level. III. Identification of soluble egg antigens recognized by cloned specific granulomagenic murine CD4 + Th 1-Type lymphocytes. $J$ Immunol 150: 1413-1421.

Cummings RD, Nyame AK 1999. Schistosome glycoconjugates. Biochim Biophys Acta 1455: 363-374.

Ferru I, Roye O, Delacre M, Auriault C, Wolowczuk I 1998. Infection of B-cell-deficient mice by the parasite Schistosoma mansoni: demonstration of the participation of B cells in granuloma modulation. Scandinavian J Immunol 48: 233-340.

Harn D, Danko K, Quinn J, Stadecker M 1989. Schisto- 
soma mansoni: the host immune response to egg antigens. I. Partial characterization $\mathrm{f}$ cellular and humoral responses to $\mathrm{pI}$ fractions of soluble egg antigens. J Immunol 142: 2061-2066.

Henderson GS, Lu X, McCurley TL, Colley DG 1992. In vivo molecular analysis of lymphokines involved in the murine immune response during Schistosoma mansoni infection. II. Quantification of IL- 4 mRNA, IFN-gamma mRNA, and IL-2 mRNA levels in the granulomatous livers, mesenteric lymph nodes, and spleens during the course of modulation. J Immunol 148: 2261-2269.

Hernandez HJ, Wang Y, Tzellas N, Stadecker MJ 1997a. Expression of class II, but not class I, major histocompatibility complex molecules is required for granuloma formation in infection with Schistosoma mansoni. Eur J Immunol 27: 1170-1176.

Hernandez HJ, Trzyna WC, Cordingley JS, Brodeur PH, Stadecker MJ 1997b. Differential antigen recognition by $\mathrm{T}$ cell populations from strains of mice developing polar forms of granulomatous inflammation in response to eggs of Schistosoma mansoni. Eur J Immunol 27: 666-670.

Hernandez HJ, Wang Y, Stadecker MJ 1997c. In infection with Schistosoma mansoni, B cells are required for $\mathrm{T}$ helper type 2 response but not for granuloma formation. J Immunol 158: 4832-4837

Hernandez HJ, Edson CM, Harn DA, Ianelli CJ, Stadecker MJ 1998. Schistosoma mansoni: genetic restriction and cytokine profile of the CD4 + T helper cell response to dominant epitope peptide of major egg antigen Sm-p40. Exp Parasitol 90: 122-130.

Hernandez HJ, Stadecker MJ 1999a. Elucidation and role of critical residues of immunodominant peptide associated with T cell-mediated parasitic disease. $J$ Immunol 163: 3877-3882.

Hernandez HJ, Sharpe AH, Stadecker MJ 1999b. Experimental murine schistosomiasis in the absence of B7 costimulatory molecules: reversal of elicited $\mathrm{T}$ cell cytokine profile and partial inhibition of egg granuloma formation. J Immunol 162: 2884-2889

Lukacs NW, Boros DL 1992. Utilization of fractionated soluble egg antigens reveals selectively modulated granulomatous and lymphokine responses during murine schistosomiasis mansoni. Infect Immun 60: 3209-3216.

Malaquias LC, Falcão PL, Silveira AM, Gazzinelli G, Prata A, Coffman RL, Pizziolo V, Souza CP, Colley DG, Correa-Oliveira R 1997. Cytokine regulation of human immune response to Schistosoma mansoni: analysis of the role of IL-4, IL-5 and IL-10 on peripheral blood mononuclear cell responses. Scandinavian J Immunol 46: 393-398.

Mathew RC, Boros DL 1986. Anti-L3T4 antibody treatment suppresses hepatic granuloma formation and abrogates antigen-induced interleukin-2 production in Schistosoma mansoni infection. Infect Immun 54: 820-826.

Mwatha JK, Kimani G, Kamau T, Mbugua GG, Ouma JH, Mumo J, Fulford AJ, Jones FM, Butterworth AE, Roberts MB, Dunne DW 1998. High levels of TNF, soluble TNF receptors, soluble ICAM-1, and IFN- gamma, but low levels of IL-5, are associated with hepatosplenic disease in human schistosomiasis mansoni. J Immunol 160: 1992-1999.

Nene V, Dunne D, Johnson K, Taylor D, Cordingley J 1986. Sequence and expression of a major egg antigen from Schistosoma mansoni. Homologies to heat shock proteins and alpha-crystallins. Mol Biochem Parasitol 21: 179-188.

Pearce E, Caspar P, Grzych J, Lewis F, Sher A 1991. Downregulation of Th1 cytokine production accompanies induction of Th2 responses by a parasitic helminth, Schistosoma mansoni. J Exp Med 173: 159-166.

Stadecker MJ, Hernandez HJ 1998. The immune response and immunopathology in infection with Schistosoma mansoni: a key role of major egg antigen Sm-p40. Parasite Immunol 20: 217-221.

Tiu WU, Davern KM, Wright MD, Board PG, Mitchell GF 1988. Molecular and serological characteristics of the glutathione S-transferases of Schistosoma japonicum and Schistosoma mansoni. Parasite Immunol 10: 693-706.

Williams DL, Asahi H, Botkin DJ, Stadecker MJ 2001. Schistosome infection stimulates host CD4+ T helper cell and B cell responses against a novel egg antigen thioredoxin peroxidase. Infect Immun 69: 1134-1141.

WynnTA, Eltoum I, Cheever AW, Lewis FA, Gause WC, Sher A 1993. Analysis of cytokine mRNA expression during primary granuloma formation induced by eggs of Schistosoma mansoni. J Immunol 151: 1430-1440.

Xu YZ, Shawar SM, Dresden MH 1990. Schistosoma mansoni: purification and characterization of a membrane-associated leucine aminopeptidase. Exp Parasitol 70: 124-133. 
New Immunogenic Egg Components • Miguel J Stadecker et al. 